Gut and Liver, Vol. 12, No. 1, January 2018, pp. 30-37

\title{
A Prospective Study of Eosinophilic Esophagitis and the Expression of Tight Junction Proteins in Patients with Gastroesophageal Reflux Disease Symptoms
}

Kenichiro Okimoto ${ }^{1}$, Makoto Arai $^{1}$, Hideaki Ishigami ${ }^{1}$, Keiko Saito ${ }^{1}$, Shoko Minemura ${ }^{1}$, Daisuke Maruoka ${ }^{1}$, Tomoaki Matsumura ${ }^{1}$, Tomoo Nakagawa ${ }^{1}$, Tatsuro Katsuno ${ }^{1}$, Masaki Suzuki ${ }^{2}$, Yukio Nakatani ${ }^{2}$, and Osamu Yokosuka ${ }^{1}$

Departments of ${ }^{1}$ Gastroenterology and ${ }^{2}$ Diagnostic Pathology, Chiba University Graduate School of Medicine, Chiba, Japan

Background/Aims: Eosinophilic esophagitis (EoE) is often erroneously diagnosed as gastroesophageal reflux disease (GERD). The aim of this study is to investigate the prevalence of EoE and the expression of tight junction (TJ) proteins in patients with GERD symptoms. Methods: One hundred patients with GERD symptoms and 10 healthy controls were prospectively studied. Sixty-two patients had symptoms refractory to proton pump inhibitors (PPI). All patients underwent esophageal biopsy. Patients were diagnosed with EoE if the number of eosinophil granulocytes per high-power field was $\geq 15$. Immunohistochemical analysis of TJ proteins (claudin-1, claudin-4, occludin, and zonula occludin-1 [ZO-1]) was performed. Results: EoE was diagnosed in six of 100 patients (6\%) with GERD symptoms and in six patients (9.7\%) of 62 patients with PPI-refractory GERD. Only one had typical EoE endoscopic findings. The proportion of ZO-1-positive cells was significantly lower in the lower than in the middle esophagus $(56.0 \% \pm 14.0 \%$ vs $66.0 \% \pm 11.5 \%, p<0.05)$. There were no significant correlations between TJ protein expression and GERD symptoms. Conclusions: The prevalence of EoE among patients with PPI-refractory GERD is approximately $10 \%$. Regardless of endoscopic findings, esophageal biopsy is crucial in diagnosing EoE. The disruption of ZO-1 expression in the lower esophagus is significantly associated with GERD symptoms. (Gut Liver 2018;12:30-37)

Key Words: Gastroesophageal reflux; Eosinophilic esophagitis; Tight junctions; Proton pump inhibitor; Zonula occludin-1

\section{INTRODUCTION}

Gastroesophageal reflux disease (GERD) is a major problem in many regions of the world. ${ }^{1}$ In 2013, the estimated prevalence of reflux esophagitis and nonerosive reflux disease in Japan was $6.8 \%$ and $15.9 \%$, respectively. ${ }^{2}$ The cause of GERD symptoms (primarily heartburn) is thought to include several factors such as acid reflux, eosinophilic esophagitis (EoE), disruption of tight junction (TJ) proteins, and cytokines. ${ }^{3-5}$

EoE is a chronic immune response to certain antigens characterized clinically by symptoms of esophageal dysfunction and histologically by eosinophil-dominated inflammation. ${ }^{6}$ Since EoE was recognized as a distinct disease entity, ${ }^{7-9}$ it has increasingly been diagnosed in Western countries. ${ }^{10-15}$ The first Japanese case of EoE was reported by Furuta et al. ${ }^{16}$ in 2006. Fujiwara et al. ${ }^{17}$ reported that of 13,634 adult patients who underwent upper endoscopy for routine screening or as a second investigation, only two $(0.015 \%)$ had EoE. Though the prevalence of EoE in Japan is still estimated to be lower than in the United States, the number of Japanese adults diagnosed with the disorder has been increasing. ${ }^{18,19}$ However, to the best of our knowledge, there are few reports evaluating the prevalence of EoE among patients with GERD symptoms.

Gastrointestinal barrier function serves to prevent unwanted substances from entering the tissue and circulation. ${ }^{20}$ In an experiment using human esophageal biopsy samples, exposure of tissue to weakly acidic and acidic solutions reduced transepithelial electrical resistance. ${ }^{4}$ Other studies have described dilation of intercellular spaces in patients with GERD, ${ }^{21}$ indicating that esophageal mucosal permeability was increased and the normal barrier function was not fully activated. The TJ is an intercellular junction crucial for epithelial adhesion and forming a tis-

Correspondence to: Makoto Arai

Department of Gastroenterology, Chiba University Graduate School of Medicine, Inohana 1-8-1, Chiba 260-8670, Japan

Tel: +81-43-226-2083, Fax: +81-43-226-2088, E-mail: araim-cib@umin.ac.jp

Received on December 14, 2016. Revised on February 19, 2017. Accepted on March 27, 2017. Published online October 17, 2017

pISSN 1976-2283 eISSN 2005-1212 https://doi.org/10.5009/gnl16600

(a) This is an Open Access article distributed under the terms of the Creative Commons Attribution Non-Commercial License (http://creativecommons.org/licenses/by-nc/4.0) which permits unrestricted non-commercial use, distribution, and reproduction in any medium, provided the original work is properly cited. 
sue barrier. ${ }^{22}$ Although changes in TJ proteins such as claudins, occludin, zonula occludin-1 (ZO-1), and E-cadherin have been reported in patients with GERD,,$^{20,23,24}$ there appears to be little consensus on the implications of this finding related to symptoms.

The aim of this study was to investigate possible associations between GERD symptoms and EoE as well as TJ proteins.

\section{MATERIALS AND METHODS}

\section{Study design and patients}

This was a prospective study conducted in Chiba University Hospital. From April 2012 to August 2016, 100 patients with GERD symptoms who had symptom scores of 8 points or more on the frequency scale for the symptoms of GERD (FSSG) ${ }^{25}$ were enrolled. The details of FSSG are shown in Fig. 1. This score has been validated in several reports. ${ }^{26,27}$ Out of these patients, we defined proton pump inhibitor (PPI)-refractory GERD as patients who had already taken PPI equal to or more than standard dose for more than 8 weeks. Of these 100 patients, 62 patients (62.0\%) were defined as having PPI-refractory GERD symptoms. Ten healthy controls who scored less than 8 points on FSSG were also enrolled in this study. Age and sex of the controls were matched with those of these 62 patients. Patients under 20 years old, who had a history of upper gastrointestinal surgery, or whose physician considered them to be ineligible to participate were excluded from this study. After selection, patients were asked about a history of allergy. Peripheral blood samples were taken to analyze the percentage of eosinophils as a fraction of total white blood cells.

Patients filled out a FSSG to evaluate the severity of GERD symptoms. FSSG comprised 12 common symptoms of GERD. Patients scored each symptom from 0 (never) to 4 (always).

This study was conducted according to the principles of the Declaration of Helsinki. All participating patients gave written informed consent. The study protocol was approved by Chiba University Institutional Review Board (IRB number: 1381) and registered (clinical registration number: UMIN000012279).

\section{Evaluation of eosinophilic infiltration and diagnosis of EoE}

All patients underwent upper endoscopy and a judgment was made as to whether their findings corresponded to reflux esophagitis or nonerosive reflux disease, according to the Los Angeles classification system. ${ }^{28}$ Regardless of the endoscopic findings, we obtained two esophageal biopsy samples from $5 \mathrm{~cm}$ (lower esophagus) and $15 \mathrm{~cm}$ (middle esophagus) above the $\mathrm{Z}$ line (four samples in total). ${ }^{29}$ Tissue samples were processed by standard protocols and stained with hematoxylin eosin. Eosinophilic infiltration was evaluated by optical microscopy (BX50; Olympus, Tokyo, Japan). Patients with PPI-refractory GERD symptoms and who had $\geq 15$ eosinophilic granulocytes per high-power field (HPF) in at least one of the four samples were diagnosed as having EoE. ${ }^{30}$ PPI-responsive esophageal eosinophilia (PPI-REE), an entity distinct from EoE according to the American College of Gastroenterology guidelines, ${ }^{30}$ was excluded from EoE.

\begin{tabular}{|c|c|c|c|c|c|c|}
\hline \multirow{2}{*}{\multicolumn{2}{|c|}{ Question }} & \multicolumn{5}{|c|}{ Frequency } \\
\hline & & \multirow{2}{*}{$\frac{\text { Never }}{0}$} & \multirow{2}{*}{$\frac{\text { Occasionally }}{1}$} & \multirow{2}{*}{$\frac{\text { Sometimes }}{2}$} & \multirow{2}{*}{$\begin{array}{c}\text { Often } \\
3\end{array}$} & \multirow{2}{*}{$\frac{\text { Always }}{4}$} \\
\hline (1) & Do you get heartburn? & & & & & \\
\hline (2) & Does your stomach get bloated? & 0 & 1 & 2 & 3 & 4 \\
\hline (3) & Does your stomach ever feel heavy after meals? & 0 & 1 & 2 & 3 & 4 \\
\hline (4) & Do you sometimes subconsciously rub your chest with your hand? & 0 & 1 & 2 & 3 & 4 \\
\hline (5) & Do you ever feel sick after meals? & 0 & 1 & 2 & 3 & 4 \\
\hline (6) & Do you get heartburn after meals? & 0 & 1 & 2 & 3 & 4 \\
\hline (7) & Do you have an unusual (e.g., burning) sensation in your throat? & 0 & 1 & 2 & 3 & 4 \\
\hline (8) & Do you feel full while eating meals? & 0 & 1 & 2 & 3 & 4 \\
\hline (9) & Do some things get stuck when you swallow? & 0 & 1 & 2 & 3 & 4 \\
\hline (10) & Do you get bitter liquid (acid) coming up into your throat? & 0 & 1 & 2 & 3 & 4 \\
\hline (11) & Do you burp a lot? & 0 & 1 & 2 & 3 & 4 \\
\hline (12) & Do you get heartburn if you bend over? & 0 & 1 & 2 & 3 & 4 \\
\hline
\end{tabular}

Fig. 1. Details of the FSSG score. Patients filled out a questionnaire to evaluate the severity of their gastroesophageal reflux disease (GERD) symptoms. This questionnaire assessed the 12 common symptoms of GERD. Patients scored each symptom from 0 (never) to 4 (always). FSSG, frequency scale for the symptoms of GERD. 
Table 1. Clinical Characteristics of 62 Patients with Reflux Symptoms Refractory to Treatment

\begin{tabular}{|c|c|c|c|}
\hline & $\begin{array}{l}\text { Patients with PPI-refractory } \\
\text { GERD symptom }(\mathrm{n}=62)\end{array}$ & $\begin{array}{l}\text { Healthy control } \\
\qquad(\mathrm{n}=10)\end{array}$ & $\mathrm{p}$-value \\
\hline Age, yr & $59.4 \pm 15.0$ & $59.4 \pm 19.0$ & $\mathrm{NS}^{*}$ \\
\hline Sex, male/female & $22 / 40$ & $3 / 7$ & $\mathrm{NS}^{\dagger}$ \\
\hline FSSG score & $19.2 \pm 7.8$ & $2.0 \pm 1.1$ & $<0.05^{*}$ \\
\hline RE/NERD & $11 / 51$ & $0 / 10$ & $\mathrm{NS}^{\dagger}$ \\
\hline Treated with $\mathrm{PPI}^{\ddagger}$ & $62(100)$ & 0 & $<0.05^{\dagger}$ \\
\hline History of allergy & $11(17.7)$ & 0 & $\mathrm{NS}^{*}$ \\
\hline Peripheral blood eosinophil, \% & $3.7 \pm 3.3$ & $3.0 \pm 2.5$ & $\mathrm{NS}^{*}$ \\
\hline
\end{tabular}

Data are presented as mean \pm SD or number $(\%)$.

PPI, proton pump inhibitor; GERD, gastroesophageal reflux disease; NS, not significant; FSSG, frequency scale for the symptoms of GERD; RE, reflux esophagitis; NERD, nonerosive reflux disease.

*Unpaired t-test; ${ }^{\dagger}$ Fisher exact test; ${ }^{\ddagger}$ Patients who had already taken PPI before enrollment.

\section{Immunohistochemistry}

To evaluate TJ proteins levels, immunohistochemical (IHC) analysis was carried out. IHC was performed on tissue samples from 80 patients with GERD symptoms whom we could obtained the agreement of their IHC analysis consecutively evaluated with endoscopy from April 2012 to September 2014 and for 10 healthy controls. The following primary antibodies were used: mouse monoclonal anti-claudin-1 (1:200 dilution, catalogue number 1440075A; Invitrogen, San Diego, CA, USA), rabbit polyclonal anti-claudin-4 (1:200, catalogue number ab53156; Abcam, Cambridge, UK), rabbit polyclonal anti-occludin (1:100, catalogue number ab31721; Abcam), and rabbit polyclonal anti-ZO-1 (1:200, catalogue number ab187012; Abcam). Positively stained cells were visualized using 3'3-diaminobenzidine and counterstained with Mayer's hematoxylin. Four HPFs per esophageal tissue sample were observed by optical microscopy and the percentage of cells in each HPF with positive staining for each TJ protein were quantified. The percentages of positively stained cells were also graded as follows. For claudin-1 and -4 , grade $1:<30 \%$, grade $2: 30 \%$ to $50 \%$, and grade 3 : $>50 \%$. For occludin and ZO-1, grade $1:<50 \%$, grade 2 : $50 \%$ to $70 \%$, and grade $3:>70 \%$. Evaluation of the tissue samples was done by investigators blinded to the diagnosis and symptom score. Percentages of positively stained cells for each TJ protein were compared in the middle and lower esophagus among the 80 patients with GERD symptoms (including all patients with EoE) and 10 healthy controls. The IHC grades and FSSG scores were evaluated for any correlation in patients with PPI-refractory GERD symptoms. Also IHC intensity of each TJ protein was classified into three groups; weak, moderate, and strong.

\section{Statistical analysis}

Patients with and without EoE were compared using Fisher exact test or unpaired t-tests, which were also used to compare age and sex between patients with PPI-refractory GERD symptoms and controls. The percentage of positively IHC stained
Table 2. A Comparison of Patients with and without EoE among 62 Patients with Reflux Symptoms Refractory to Treatment

\begin{tabular}{lccc}
\hline & $\begin{array}{c}\text { EoE } \\
(\mathrm{n}=6)\end{array}$ & $\begin{array}{c}\text { non-EoE } \\
(\mathrm{n}=56)\end{array}$ & $\mathrm{p}$-value \\
\hline Age, yr & $64.2 \pm 11.0$ & $58.9 \pm 15.7$ & $\mathrm{NS}^{*}$ \\
Sex, male/female & $2 / 4$ & $20 / 36$ & $\mathrm{NS}^{\dagger}$ \\
FSSG score & $18.5 \pm 7.9$ & $19.2 \pm 8.0$ & $\mathrm{NS}^{*}$ \\
RE/NERD & $1 / 5$ & $10 / 46$ & $\mathrm{NS}^{\dagger}$ \\
History of allergy & $2(33.3)$ & $10(17.9)$ & $\mathrm{NS}^{\dagger}$ \\
Peripheral blood eosinophil, \% & $4.4 \pm 2.8$ & $3.6 \pm 3.6$ & $\mathrm{NS}^{*}$ \\
\hline
\end{tabular}

Data are presented as mean \pm SD or number (\%).

EoE, eosinophilic esophagitis; NS, not significant; FSSG, frequency scale for the symptoms of gastroesophageal reflux disease; RE, reflux esophagitis; NERD, nonerosive reflux disease.

*Unpaired t-test; ${ }^{\dagger}$ Fisher exact test.

cells in the middle and lower esophagus as well as inter-group analysis were analyzed using unpaired t-tests. IHC intensity was analyzed using chi-square test. Potential correlations between the grades of IHC staining for each TJ protein and the FSSG scores were evaluated using Pearson correlation. A p-value of $<0.05$ was considered to be statistically significant. All statistical analyses were performed using SPSS version 22.0 (IBM Corp., Armonk, NY, USA).

\section{RESULTS}

\section{Characteristics of patients with PPI-refractory GERD}

Characteristics of the 62 patients with PPI-refractory GERD symptoms and 10 healthy controls are shown in Table 1 . There were no significant differences between the two groups other than the use of PPIs. Out of 62 patients, six patients (9.7\%) were diagnosed as EoE. These patients had already taken PPI for more than 8 weeks. As their esophageal biopsy and FSSG score measurement were performed after 8-week PPI trial, these patients were not PPI-REE. One in 100 patients with GERD symptoms 
Table 3. Clinical Characteristics of the Individual Patients Included in the EoE Group

\begin{tabular}{|c|c|c|c|c|c|c|c|c|}
\hline & \multirow{2}{*}{ Sex } & \multirow{2}{*}{ Age, yr } & \multirow{2}{*}{ Chief complaint } & \multirow{2}{*}{ FSSG score } & \multirow{2}{*}{ Eosinophil/HPF } & \multirow{2}{*}{ Endoscopic findings } & \multicolumn{2}{|c|}{ PPI-trial* ${ }^{*}$} \\
\hline & & & & & & & Dose of PPI/day & Duration period, wk \\
\hline 1 & M & 72 & Dysphagia & 16 & 18 & - & Rabeprazole $20 \mathrm{mg}$ & 10 \\
\hline 2 & M & 69 & Heartburn & 10 & 20 & - & Rabeprazole $20 \mathrm{mg}$ & 16 \\
\hline 3 & $\mathrm{~F}$ & 56 & Epigastralgia & 11 & 40 & - & Rabeprazole $20 \mathrm{mg}$ & 12 \\
\hline 4 & $\mathrm{~F}$ & 67 & Dysphagia & 22 & 30 & - & Lansoprazole $15 \mathrm{mg}$ & 8 \\
\hline 5 & F & 46 & Heartburn & 21 & 47 & Longitudinal furrows & Rabeprazole $20 \mathrm{mg}$ & 8 \\
\hline 6 & $\mathrm{~F}$ & 75 & Heartburn & 31 & 40 & - & Rabeprazole $20 \mathrm{mg}$ & 40 \\
\hline
\end{tabular}

EoE, eosinophilic esophagitis; FSSG, frequency scale for the symptoms of gastroesophageal reflux disease; HPF, high-power field; PPI, proton pump inhibitor; $\mathrm{M}$, male; $\mathrm{F}$, female.

*All patients in this table had already taken PPI for more than 8 weeks before enrollment in this study.
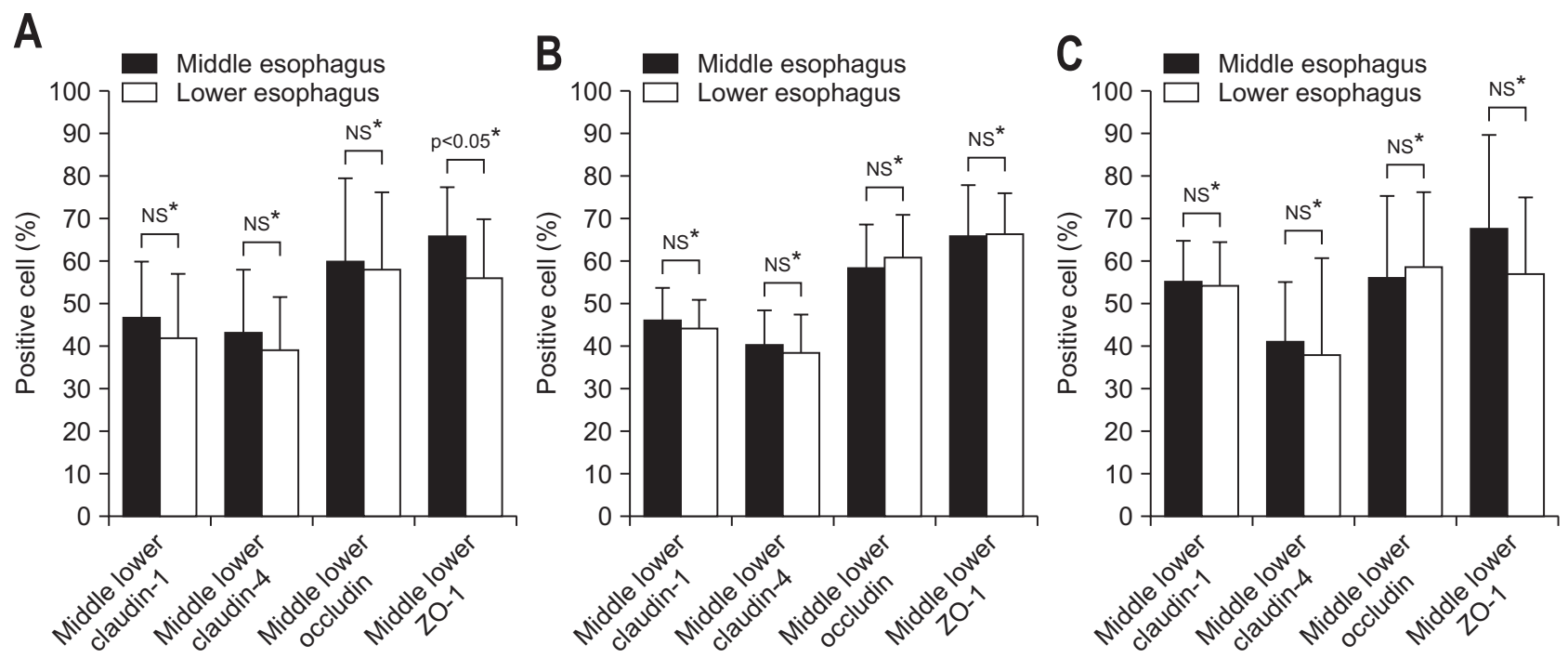

Fig. 2. A comparison of the percentages of cells that stained positively for each tight junction protein in the middle and lower esophagus. (A) Patients with proton pump inhibitor refractory gastroesophageal reflux disease symptoms $(n=62)$. There were no significant differences in the proportions of cells positive for claudin-1, claudin-4, or occludin between the middle and lower esophagus. The percentage of zonula occludin-1 (ZO-1)-positive cells, however, was significantly lower in the lower than in the middle esophagus $(56.0 \% \pm 14.0 \% \mathrm{vs} 66.0 \% \pm 11.5 \%$, $\mathrm{p}<0.05)$. (B) Healthy controls $(n=10)$. There were no significant differences between the proportion of cells positive for claudin-1, claudin-4, occludin, or Z0-1 in the middle and lower esophagus. (C) Patients with eosinophilic esophagitis (EoE) $(n=6)$. There were no significant differences in the proportion of cells positive for claudin-1, claudin-4, occludin, or ZO-1 between the middle and lower esophagus.

NS, not significant. *Unpaired t-test.

was diagnosed as PPI-REE. This patient was excluded from EoE.

\section{Comparison of patients with and without EoE}

Out of 62 patients with PPI-refractory GERD, six (9.7\%) were diagnosed with EoE (Table 2). There were no significant differences between the two groups with respect to age, sex, FSSG score, the proportions of reflux esophagitis or nonerosive reflux disease, blood eosinophil counts, or a history of allergy. The details of six EoE patients were shown in Table 3.

\section{IHC analysis of TJ proteins in the middle and lower esophagus}

The percentages of positively staining cells for each TJ protein were compared between the middle and lower esophagus in the 62 patients with PPI-refractory GERD symptoms (Fig. 2A). The percentage of claudin-1-positive cells was $46.9 \% \pm 13.1 \%$ in the middle and $42.0 \% \pm 15.2 \%$ in the lower esophagus. The percentage of claudin-4-positive cells was $43.6 \% \pm 14.5 \%$ in the middle and $39.1 \% \pm 12.7 \%$ in the lower esophagus. The percentage of occludin-positive cells was $60.2 \% \pm 19.3 \%$ in the middle and $58.2 \% \pm 18.2 \%$ in the lower esophagus. There were no significant differences between the middle and lower esophagus for these three TJ proteins. The percentage of cells staining positively for ZO-1 in the lower esophagus was significantly lower than that in the middle esophagus $(56.0 \% \pm 14.0 \%$ vs $66.0 \% \pm 11.5 \%, p<0.05)$. Similar data are shown in Fig. $2 B$ for controls and Fig. 2C for patients with EoE. The percentages of claudin-1-positive cells were $45.9 \% \pm 7.7 \%$ and $55.5 \% \pm 14.1 \%$ 
in the middle and $44.2 \% \pm 6.7 \%$ and $54.3 \% \pm 22.8 \%$ in the lower esophagus, respectively. The percentages of claudin-4-positive cells were $40.6 \% \pm 7.8 \%$ and $41.4 \pm 19.4 \%$ in the middle $38.6 \% \pm 9.1 \%$ and $38.1 \% \pm 18.0 \%$ in the lower esophagus, respectively. The percentage of occludin-positive cells was 58.7\% $9.8 \%$ and $56.4 \% \pm 22.0 \%$ in the middle and $60.7 \% \pm 10.0 \%$ and $58.7 \% \pm 18.3 \%$ in the lower esophagus, respectively. The percentages of Z0-1-positive cells were $65.9 \% \pm 12.1 \%$ and $68.1 \% \pm 9.5 \%$ in the middle and $66.2 \% \pm 9.5 \%$ and $57.1 \% \pm 10.4 \%$ in the lower esophagus, respectively. There were no significant differences in these two groups between the middle and lower esophagus for all TJ proteins. The expression of $\mathrm{ZO}-1$ in the lower esophagus was almost the same as in the middle esophagus in the healthy controls and patients with EoE, in contrast to the differential expression pattern of ZO-1 observed in patients with PPI-refractory GERD symptoms. We also performed intergroup (PPI-refractory GERD, EoE, and healthy control) comparison. There was no significant difference in staining middle and lower esophagus between any of these two groups as for the expression of each $\mathrm{TJ}$ protein (unpaired t-test).

IHC intensity analysis was shown in Fig. 3. The ratio of weak:middle:strong intensity in PPI-refractory GERD, EoE, and healthy control was 48.4\%:41.9\%:9.7\%, 50\%:50\%:0\%, and 60\%:30\%:0\% in claudin-1; 64.5\%:30.6\%:4.9\%, 50\%:33.3\%: 16.7\%, and 50\%:40\%:10\% in claudin-4; 61.3\%:35.5\%:3.2\%, 50\%:33.3\%:16.7\%, and 60\%:30\%:10\% in occludin; 16.1\%: 64.5\%:19.4\%, 16.7\%:66.7\%:16.7\%, and 20\%:60\%:20\% in Z0-1. There were no significant difference among those three groups as for any TJ proteins (chi-square test).

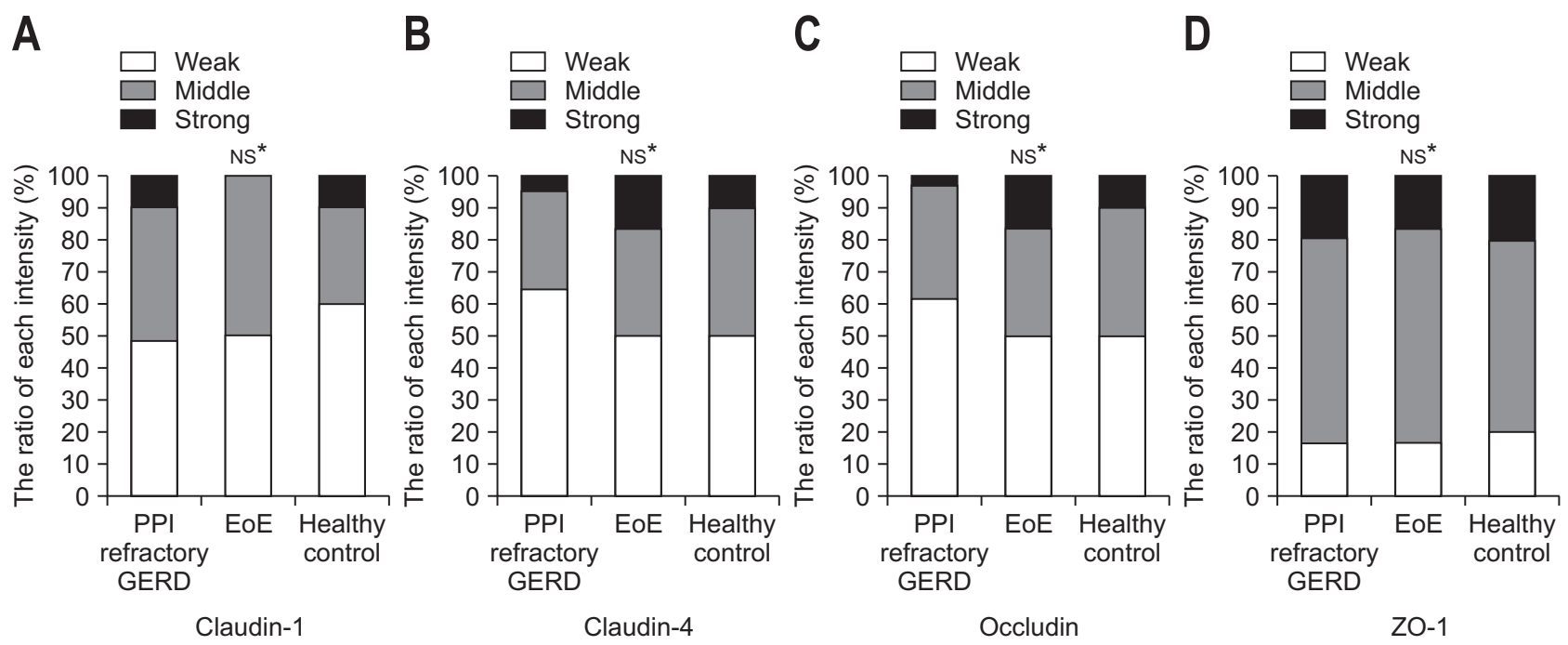

Fig. 3. The immunohistochemical intensity analysis among proton pump inhibitor (PPI)-refractory gastroesophageal reflux disease (GERD), eosinophilic esophagitis (EoE), and healthy control individuals. White, gray, and black columns show the ratios of weak, middle, and strong intensity for each tight junction protein.

NS, not significant; ZO-1, zonula occludin-1. *Chi-square test.

A

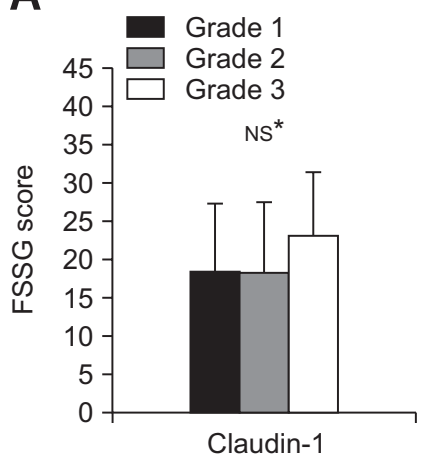

B

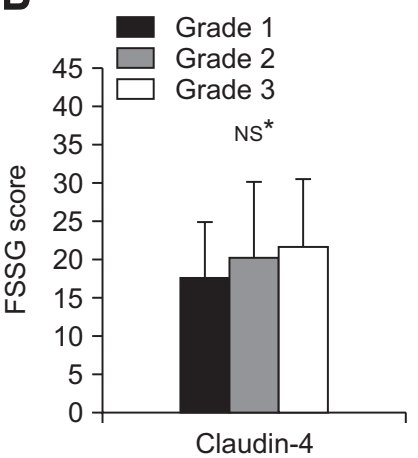

C

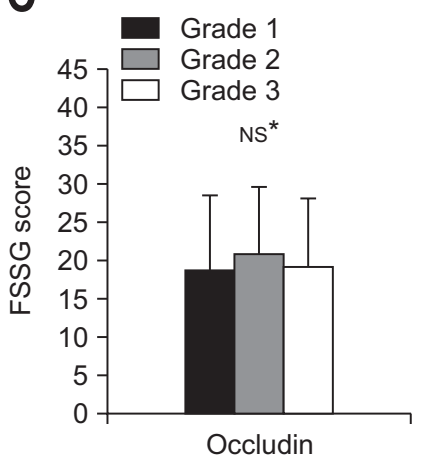

D

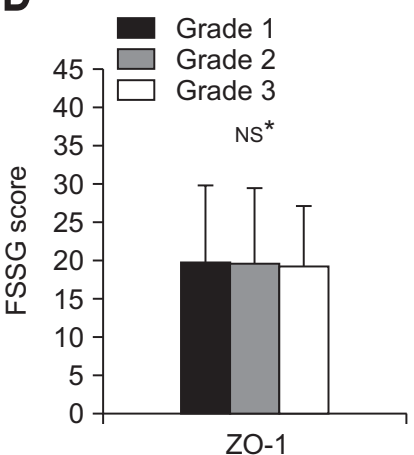

Fig. 4. An assessment using Pearson correlation between the tight junction (TJ) protein immunohistochemical (IHC) staining grades and the FSSG scores in 80 patients who underwent endoscopies. There were no significant correlations between symptoms and IHC staining grades for any TJ proteins (IHC staining was graded as follows: claudin-1 and -4: grade 1, <30\%; grade 2, 30\%-50\%; grade 3, >50\%; occludin and Z0-1: grade 1, $<50 \%$; grade 2, 50\%-70\%; grade 3, >70\%).

FSSG, frequency scale for the symptoms of gastroesophageal reflux disease; NS, not significant; Z0-1, zonula occludin-1. 


\section{Correlation between IHC grades and FSSG score}

Potential correlations between IHC staining grades and severity of GERD symptoms were analyzed in 80 patients (Fig. 4). For claudin-1, FSSG scores for IHC grade 1 staining were $18.5 \pm 8.7$ (six patients); grade 2, 18.4 \pm 9.2 (30 patients); and grade 3, $23.3 \pm 8.1$ ( 15 patients) $(r=0.08, p=0.5)$. For claudin-4, FSSG scores for IHC grade 1 staining were $17.5 \pm 7.3$ (13 patients); grade 2, $20.2 \pm 9.8$ (26 patients); and grade $3,21.7 \pm 8.8$ (12 patients) ( $\mathrm{r}=0.08, \mathrm{p}=0.49)$. For occludin, FSSG scores for IHC grade 1 staining were $18.8 \pm 9.8$ (17 patients); grade 2 , 21.2 \pm 8.6 (18 patients); and grade $3,19.5 \pm 8.7$ (16 patients) ( $\mathrm{r}=0.004, \mathrm{p}=0.97$ ). For ZO-1, FSSG scores for IHC grade 1 staining were $19.9 \pm 10.0$ (eight patients); grade 2, 19.8 \pm 9.7 (33 patients); and grade 3, $19.5 \pm 7.8$ (10 patients) $(\mathrm{r}=-0.07, \mathrm{p}=0.56)$. No significant correlations were identified between FSSG scores and IHC staining grades for any TJ proteins.

\section{DISCUSSION}

The results of this study showed that approximately 10\% patients with PPI-refractory GERD symptoms had EoE. Most patients with EoE, however, did not have typical endoscopic findings of that disorder.

The reported proportions of patients with typical endoscopic findings for EoE in a study in Japan were 35\% with longitudinal furrows, 23\% with white plaques, and 19\% with multiple concentric rings. ${ }^{19}$ In about $20 \%$ to $40 \%$ of patients with EoE, none of these findings were detected. ${ }^{19,29}$ This was true of even a higher proportion of patients with EoE in our study, with five of six having no typical endoscopic findings. PPI has been reported to improve EoE endoscopic findings. ${ }^{31}$ The high frequency of PPI use in our subjects might thus help explain the paucity of typical findings. In Japan, the prevalence of EoE is thought to be relatively low, but this perception might change if more patients undergoing endoscopy had esophageal biopsy with appropriate evaluation for eosinophilic infiltrates. If the disease is diagnosed based only on the macroscopic endoscopic appearance, we suspect a substantial number of patients with EoE are misdiagnosed as having PPI-refractory GERD. These two conditions must be distinguished, as the treatments differ. Topical steroids are sometimes effective for EoE, ${ }^{32}$ while PPI and $\mathrm{H} 2$ receptor antagonists are generally favored for the treatment of GERD. ${ }^{33}$ Our findings suggest that, in order to diagnose EoE correctly, esophageal biopsy should be performed regardless of the endoscopic findings. The importance of esophageal biopsy with endoscopy for the patients with incomplete PPI response to find out EoE patients was also previously reported. ${ }^{34}$ Miller et al. ${ }^{35}$ reported that esophageal biopsy for EoE appears to be cost effective when the prevalence of EoE is $8 \%$ or greater. Our findings of unsuspected EoE favor this recommendation.

TJs are intracellular junctions consisting of four types of transmembrane proteins, including the claudin family, occludin, junctional adhesion molecules, and tricellulin, as well as numerous cytosolic proteins. ${ }^{36}$ Claudins are crucial for the barrier function of TJs. Decreased expression of claudin-1 and -4 contributes to increased permeability of the gastrointestinal epithelium in GERD. ${ }^{20}$ Occludin is also a key protein for proper TJ function in various tissues. ZO-1 is classified as a cytosolic proteins to which claudins and occludin bind. ${ }^{36}$ The selection of $\mathrm{TJ}$ proteins in this study was based on these characteristics. We found the proportion of Z0-1-positive cells in the lower esophagus was significantly lower than that in the middle esophagus in patients with PPI-refractory GERD symptoms. Tack et al. ${ }^{37}$ reported that bile salt reflux was observed in 38\% of patients with PPI-refractory GERD. In addition to gastric acid, bile salts and trypsin are reported to have the potential to disrupt the esophageal barrier function by modulating TJ proteins. ${ }^{20}$ The lower ZO-1 expression found in our patients might be attributed not only to acid reflux but also to bile salt reflux. The contrasting finding of no such difference between healthy controls and the patients with EoE suggests that TJ proteins alone may not play an important role in the pathophysiology of EoE. FSSG scores were not significantly correlated with the expression of TJ proteins, including Z0-1. However, in a previous report, Z0-1 gene expression was significantly correlated with transepithelial electrical resistance while claudin-1 and occludin were not. ${ }^{28}$ The function of ZO-1 needs further analysis.

This study has some limitations. Firstly, the number of patients with PPI-refractory GERD symptoms was small. Secondly, evaluation of TJ protein expression was based only on IHC analysis. To further clarify the role of TJ proteins, methods in addition to IHC should be used. However, there have been few prospective reports regarding the prevalence of EoE and esophageal expression of TJ proteins in patients with PPI-refractory GERD symptoms. In this respect, this study is novel. It will be useful to investigate a larger population, including healthy controls, to further confirm our results.

In conclusion, the prevalence of EoE among patients with PPI-refractory GERD symptoms 9.7\%, which was surprisingly high. Esophageal biopsy is essential to diagnose EoE in such patients, as the endoscopic findings may not be typical of EoE. In patients with GERD symptoms, Z0-1 expression in the lower esophagus was significantly reduced compared with that in the middle esophagus. This disease-associated change might be important result to consider in future research to gain a fuller understanding of the role of TJ proteins in patients with PPIrefractory GERD symptoms.

\section{CONFLICTS OF INTEREST}

No potential conflict of interest relevant to this article was reported. 


\section{ACKNOWLEDGEMENTS}

We wish to thank Saegusa for the support of pathological investigation.

Author contribution: K.O. wrote the paper; M.A. designed research and wrote the paper; H.I., K.S., S.M., D.M., T.M., T.N., T.K. performed upper endoscopy; M.S., Y.N. supervised pathological examination; O.Y. analyzed data.

\section{REFERENCES}

1. Kang JY. Systematic review: geographical and ethnic differences in gastro-oesophageal reflux disease. Aliment Pharmacol Ther 2004;20:705-717.

2. Minatsuki C, Yamamichi N, Shimamoto T, et al. Background factors of reflux esophagitis and non-erosive reflux disease: a cross-sectional study of 10,837 subjects in Japan. PLoS One 2013;8:e69891.

3. Furuta GT, Liacouras CA, Collins MH, et al. Eosinophilic esophagitis in children and adults: a systematic review and consensus recommendations for diagnosis and treatment. Gastroenterology 2007;133:1342-1363.

4. Woodland P, Lee C, Duraisamy Y, Farré R, Dettmar P, Sifrim D. Assessment and protection of esophageal mucosal integrity in patients with heartburn without esophagitis. Am J Gastroenterol 2013;108:535-543.

5. Dunbar KB, Agoston AT, Odze RD, et al. Association of acute gastroesophageal reflux disease with esophageal histologic changes. JAMA 2016;315:2104-2112.

6. Liacouras CA, Furuta GT, Hirano I, et al. Eosinophilic esophagitis: updated consensus recommendations for children and adults. J Allergy Clin Immunol 2011;128:3-20.e6.

7. Liacouras CA, Markowitz JE. Eosinophilic esophagitis: a subset of eosinophilic gastroenteritis. Curr Gastroenterol Rep 1999;1:253258.

8. Borda F, Jiménez FJ, Martínez Peñuela JM, Echarri A, Martín Granizo I, Aznarez R. Eosinophilic esophagitis: an underdiagnosed entity? Rev Esp Enferm Dig 1996;88:701-704.

9. Attwood SE, Smyrk TC, Demeester TR, Jones JB. Esophageal eosinophilia with dysphagia: a distinct clinicopathologic syndrome. Dig Dis Sci 1993;38:109-116.

10. Arora AS, Yamazaki K. Eosinophilic esophagitis: asthma of the esophagus? Clin Gastroenterol Hepatol 2004;2:523-530.

11. Potter JW, Saeian K, Staff D, et al. Eosinophilic esophagitis in adults: an emerging problem with unique esophageal features. Gastrointest Endosc 2004;59:355-361.

12. Liacouras CA, Spergel JM, Ruchelli E, et al. Eosinophilic esophagitis: a 10-year experience in 381 children. Clin Gastroenterol Hepatol 2005;3:1198-1206.

13. Straumann A, Spichtin HP, Grize L, Bucher KA, Beglinger C, Simon HU. Natural history of primary eosinophilic esophagitis: a follow-up of 30 adult patients for up to 11.5 years. Gastroenterol- ogy 2003;125:1660-1669.

14. Prasad GA, Alexander JA, Schleck CD, et al. Epidemiology of eosinophilic esophagitis over three decades in Olmsted County, Minnesota. Clin Gastroenterol Hepatol 2009;7:1055-1061.

15. Dellon ES, Gibbs WB, Fritchie KJ, et al. Clinical, endoscopic, and histologic findings distinguish eosinophilic esophagitis from gastroesophageal reflux disease. Clin Gastroenterol Hepatol 2009;7:1305-1313.

16. Furuta K, Adachi K, Kowari K, et al. A Japanese case of eosinophilic esophagitis. J Gastroenterol 2006;41:706-710.

17. Fujiwara Y, Sugawa T, Tanaka F, et al. A multicenter study on the prevalence of eosinophilic esophagitis and PPI-responsive esophageal eosinophilic infiltration. Intern Med 2012;51:3235-3239.

18. Abe Y, Iijima K, Ohara S, et al. A Japanese case series of 12 patients with esophageal eosinophilia. J Gastroenterol 2011;46:2530.

19. Kinoshita Y, Furuta K, Ishimaura N, et al. Clinical characteristics of Japanese patients with eosinophilic esophagitis and eosinophilic gastroenteritis. J Gastroenterol 2013;48:333-339.

20. Björkman EV, Edebo A, Oltean M, Casselbrant A. Esophageal barrier function and tight junction expression in healthy subjects and patients with gastroesophageal reflux disease: functionality of esophageal mucosa exposed to bile salt and trypsin in vitro. Scand J Gastroenterol 2013;48:1118-1126.

21. Calabrese C, Fabbri A, Bortolotti M, et al. Dilated intercellular spaces as a marker of oesophageal damage: comparative results in gastro-oesophageal reflux disease with or without bile reflux. Aliment Pharmacol Ther 2003;18:525-532.

22. Niessen CM. Tight junctions/adherens junctions: basic structure and function. J Invest Dermatol 2007;127:2525-2532.

23. Weijenborg PW, Smout AJ, Verseijden C, et al. Hypersensitivity to acid is associated with impaired esophageal mucosal integrity in patients with gastroesophageal reflux disease with and without esophagitis. Am J Physiol Gastrointest Liver Physiol 2014;307:G323-G329.

24. Mönkemüller K, Wex T, Kuester D, et al. Role of tight junction proteins in gastroesophageal reflux disease. BMC Gastroenterol 2012;12:128.

25. Kusano M, Shimoyama Y, Sugimoto S, et al. Development and evaluation of FSSG: frequency scale for the symptoms of GERD. J Gastroenterol 2004;39:888-891.

26. Izawa S, Funaki Y, Iida A, et al. The role of gastroesophageal reflux in relation to symptom onset in patients with proton pump inhibitor-refractory nonerosive reflux disease accompanied by an underlying esophageal motor disorder. Digestion 2014;89:61-67.

27. Tominaga K, Iwakiri R, Fujimoto K, et al. Rikkunshito improves symptoms in PPI-refractory GERD patients: a prospective, randomized, multicenter trial in Japan. J Gastroenterol 2012;47:284292.

28. Lundell LR, Dent J, Bennett JR, et al. Endoscopic assessment of oesophagitis: clinical and functional correlates and further validation of the Los Angeles classification. Gut 1999;45:172-180. 
29. Mackenzie SH, Go M, Chadwick B, et al. Eosinophilic oesophagitis in patients presenting with dysphagia: a prospective analysis. Aliment Pharmacol Ther 2008;28:1140-1146.

30. Dellon ES, Gonsalves N, Hirano I, et al. ACG clinical guideline: evidenced based approach to the diagnosis and management of esophageal eosinophilia and eosinophilic esophagitis (EoE). Am J Gastroenterol 2013;108:679-692.

31. Ngo P, Furuta GT, Antonioli DA, Fox VL. Eosinophils in the esophagus: peptic or allergic eosinophilic esophagitis? Case series of three patients with esophageal eosinophilia. Am J Gastroenterol 2006;101:1666-1670.

32. Alexander JA, Jung KW, Arora AS, et al. Swallowed fluticasone improves histologic but not symptomatic response of adults with eosinophilic esophagitis. Clin Gastroenterol Hepatol 2012;10:742749.e1.

33. Katz PO, Gerson LB, Vela MF. Guidelines for the diagnosis and management of gastroesophageal reflux disease. Am J Gastroenterol 2013;108:308-328.

34. Kahrilas PJ, Boeckxstaens G, Smout AJ. Management of the patient with incomplete response to PPI therapy. Best Pract Res Clin Gastroenterol 2013;27:401-414.

35. Miller SM, Goldstein JL, Gerson LB. Cost-effectiveness model of endoscopic biopsy for eosinophilic esophagitis in patients with refractory GERD. Am J Gastroenterol 2011;106:1439-1445.

36. Ichikawa-Tomikawa N, Sugimoto K, Satohisa S, Nishiura K, Chiba $\mathrm{H}$. Possible involvement of tight junctions, extracellular matrix and nuclear receptors in epithelial differentiation. J Biomed Biotechnol 2011;2011:253048.

37. Tack J, Koek G, Demedts I, Sifrim D, Janssens J. Gastroesophageal reflux disease poorly responsive to single-dose proton pump inhibitors in patients without Barrett's esophagus: acid reflux, bile reflux, or both? Am J Gastroenterol 2004;99:981-988. 